\title{
Un modelo conceptual sobre el funcionamiento de los ríos mediterráneos sometidos a perturbaciones naturales (riadas y sequías)
}

\author{
Ma Rosario Vidal-Abarca Gutiérrez * y Mª Luisa Suárez Alonso
}

Departamento de Ecología e Hidrología. Facultad de Biología. Universidad de Murcia. Campus de Espinardo 30100 Murcia. España

* Corresponding author: charyvag@um.es

\begin{abstract}
A conceptual model on the function of Mediterranean rivers subjected to natural disturbances (flash-floods and droughts)

The Mediterranean regions are prone to suffer extraordinary flash-floods and prolonged droughts. Between these extreme events, there are small floods and droughts that are more common and more predictable. These smaller floods and droughts, along with the others mentioned before generate an extraordinarily complex temporal hydrological picture whose affects have been partly described by different authors, including those affecting the human population.

The information compiled during nearly two decades by the group of Ecology of Continental Waters of the University of Murcia, about the effects of these events on rivers of the Segura River Basin, allows for elaborating a conceptual model about the function of several physical, physico-chemical, and biological parameters in rivers in the southwest, in particular and Mediterranean rivers in general.
\end{abstract}

Key words: Flash-floods, droughts, floods, droughts, Mediterranean rivers, conceptual model.

\section{RESUMEN}

Un modelo conceptual sobre el funcionamiento de los ríos mediterráneos sometidos a perturbaciones naturales (riadas y sequías)

Las regiones mediterráneas son proclives a sufrir avenidas extraordinarias y sequías prolongadas. Entre estos eventos extremos, se sitúan las crecidas y estiajes más habituales y predecibles que, junto a los anteriores configuran un cuadro hidrologico temporal extraordinariamente complejo cuyos efectos han sido descritos parcialmente por diferentes autores, incluidos aquellos que afectan a la población humana.

La información recogida durante casi dos décadas por el Grupo de Ecología de Aguas Continentales de la Universidad de Murcia, sobre los efectos de estos eventos en ríos de la Cuenca del Segura, permite elaborar un modelo conceptual sobre el funcionamiento de distintos parámetros físicos, físico-químicos y biológicos en ríos del sureste, en particular y mediterráneos, en general.

Palabras clave: Avenidas, sequías, crecidas, estiajes, ríos mediterráneos, modelo conceptual.

\section{INTRODUCCIÓN}

La Limnología tiene varios temas "pendientes", entre los que destaca el estudio de los ecosistemas acuáticos de las regiones áridas y semiáridas. Estas regiones se distribuyen en todo el planeta ocupando un $68 \%$ del territorio (Davies et al.,
1994), pero solo recientemente están recibiendo atención por parte de científicos, investigadores y público en general, probablemente como respuesta a la amenaza que supone el cambio global. En España, la región árida y semiárida se sitúa, fundamentalmente, en la parte central de la depresión del Ebro y el sureste peninsular (García 

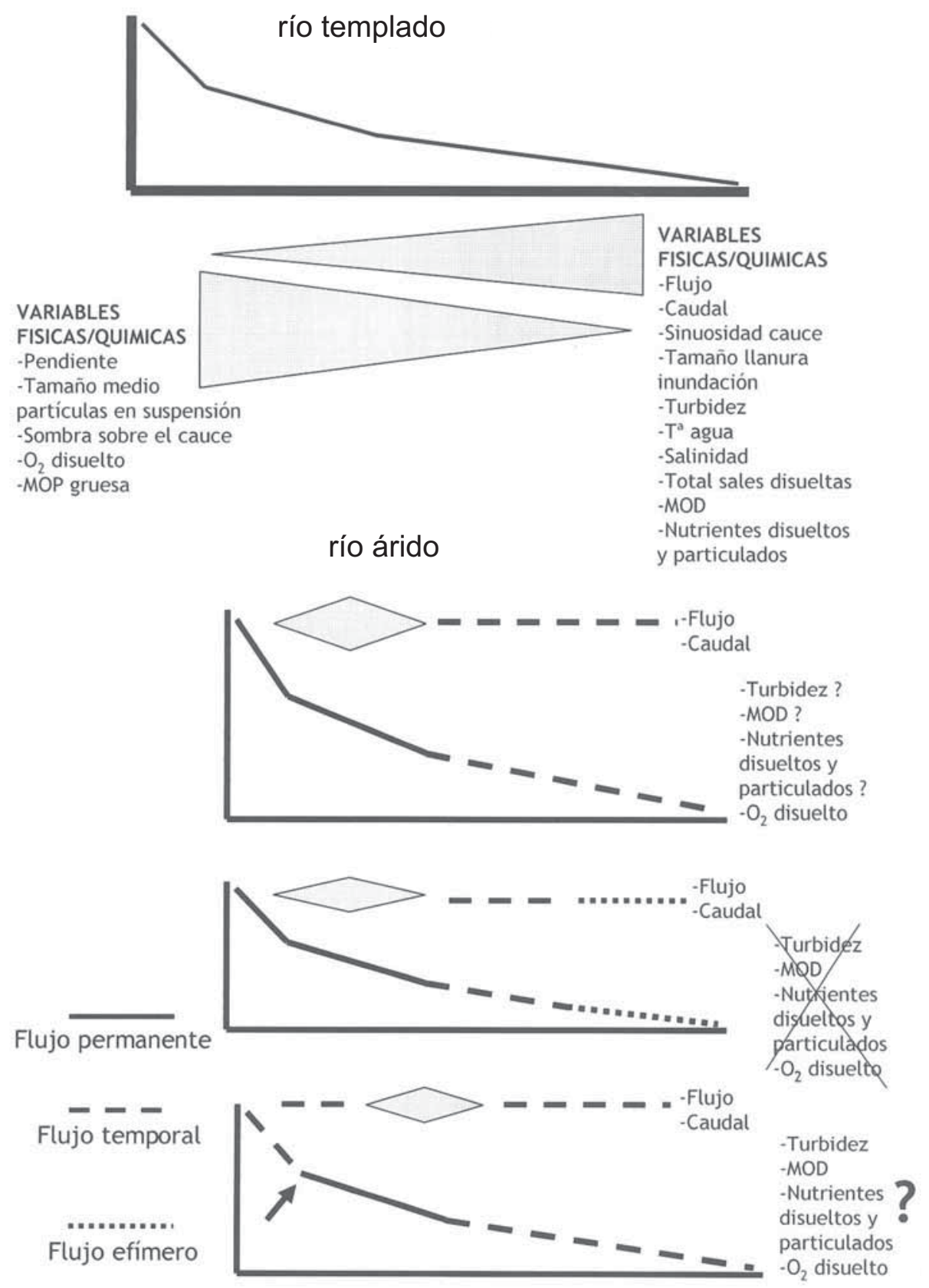

Figura 1. Esquema simplificado de la variación desde cabecera a desembocadura de distintos parámetros físicos y químicos en ríos de la zona templada y de la zona árida. Los triángulos oscuros marcan el sentido del cambio. Simplified diagram of the variation of different physical and chemical parameters from head to mouth for rivers in temperate and arid zones. Dark triangles show direction of change.

de Pedraza, 1989). Ambientalmente se caracterizan porque el balance de agua es negativo debido a condiciones o circunstancias climáticas es- tresantes (escasas precipitaciones y muy variables en el espacio y en el tiempo, alta radiación solar, escasa infiltración del agua en el suelo, 
etc). La extrema variabilidad en los componentes abiótico y bióticos, es decir una gran heterogeneidad espacial y temporal es, probablemente el principal rasgo de los ecosistemas de las regiones semiáridas (Likens, 1999).

Recientemente empiezan a aparecer algunos datos en los libros de texto en los que se destacan aspectos descriptivos y funcionales de los ecosistemas acuáticos de estas regiones (Davies et al., 1994; Giller \& Malmqvist, 1998; Cushing \& Allan, 2001; Dodds, 2002). Su importancia radica en que estos ecosistemas pueden ser los "testigos" actuales de los efectos que el cambio climático está provocando en la Tierra (Firth \& Fisher, 1992). Uno de estos efectos parece afectar de forma extrema a la hidrología en dos sentidos: aumentando la temporalidad y la intermitencia del agua, de manera que los ríos y otros ecosistemas acuáticos de las tierras áridas y semiáridas, se convierten en verdaderos laboratorios naturales (Grimm \& Fisher, 1992; Lake, 2000) donde ensayar y analizar las consecuencias que, sobre el ambiente físico, químico, y biológico tiene el cambio global.

\section{El marco teórico}

Desde hace varias décadas, se han generado distintos modelos teóricos con diferente complejidad que intentan explicar el funcionamiento de los ecosistemas acuáticos, en general y de los ríos, en particular (The River continuum concept, Vannote et al., 1980; The serial discontinuity concept, Ward \& Standford, 1983; The flood pulse concept, Junk et al., 1989; The hiporheic corridor, Standford \& Ward, 1993; A telescoping ecosystem model, Fisher et al., 1998). El problema es que al verificarlos, casi ninguno se ajusta a lo que ocurre en los ríos de las tierras áridas y semiáridas. En efecto, en una simplificación esquemática, como la que se presenta en la figura 1, se puede observar el sentido de variación, desde cabecera a desembocadura, de distintos parámetros físicos, químicos y ambientales que caracterizan a un río de la región templada y que explicaría buena parte de la estructura y funcionamiento de estos sistemas. Sin embargo, simplemente las distintas manifestaciones espaciales del flujo de agua (permanente, temporal o efímero), en ríos de las regiones áridas, y su secuencia a lo largo del eje longitudinal, crea muchas incertidumbres sobre el sentido del cambio de algunas de estas variables.

El origen de la singularidad de los ríos de ambientes áridos y semiáridos está en la extraordinaria variabilidad hidrológica que exhiben (Vidal-Abarca, 1990; Vidal-Abarca et al., 1992; 2004) y que, como primera aproximación permite clasificarlos como permanentes, temporales y efímeros, según el tiempo de permanencia del agua en el cauce. En la literatura es posible encontrar muchas otras clasificaciones diferentes (por ejemplo, Paijmans, 1985 in Boulton \& Brock, 1999; Comin \& Williams, 1994; Poff, 1996; Uys \& O’Keeffe, 1997).

Pero el principal problema viene cuando se hace intervenir la escala temporal en toda su dimensión. La variabilidad hidrológica, entonces, se manifiesta desde una escala diaria hasta la consideración de una variabilidad plurianual de extraordinaria complejidad (Vidal-Abarca et $a l .$, 2004). El origen de esta variabilidad es indudablemente el clima. En concreto el régimen de precipitaciones unido a la tasa de evaporación del lugar (Boulton, 2000). Las perturbaciones hidrológicas naturales marcan definitivamente este esquema hidrológico (Vidal-Abarca et al., 1992; 1995). Como perturbaciones extremas se reconocen las avenidas de agua y las sequías (Fisher \& Grimm, 1988; Suárez et al., 1993; 1995; Molina et al., 1994), frente a las crecidas producidas tras las lluvias de otoño, principalmente, y los estiajes típicos del verano mediterráneo (Gasith \& Resh, 1999; Lake, 2003), que se deberían considerar como parte de la variabilidad natural de la hidrología de estos ecosistemas (Vidal-Abarca et al., 2004).

A escala anual, algunos autores han descrito distintos modelos hidrológicos. Así, Boulton y Lake (1990) definieron cinco fases hidrológicas (pre-flujo, flujo temprano, flujo principal, disminución del flujo y post-flujo), en dos ríos intermitentes australianos, cada una de ellas caracterizada por una combinación compleja de distintas variables físicas y químicas. Más tarde, Stanley y Fisher (1992) lo simplifican a tres fases hidrológicas diferentes (Fase I: pérdida progresiva 
del flujo de agua; Fase II: sequía o ausencia de agua superficial; Fase III: restitución del flujo de agua), en función de los cambios detectados para distintas variables biológicas, interpretados en términos de la teoría de la estabilidad (cada fase influye sobre la abundancia, distribución y ciclo de vida característico de los organismos del río).

Sin embargo, el rango de variación es mucho mayor de lo que se observa en estos modelos. La certeza de que dos años hidrológicos consecutivos sean iguales, o al menos similares, es prácticamente nula, lo cual complica enormemente la posibilidad de modelizar cualquier aspecto del medio físico, químico, biológico, metabólico o funcional en estos sistemas. Así pues, nuestra hipótesis de partida es que si la variabilidad climática (sobre todo del régimen de precipitaciones) en las regiones áridas y semiáridas es tan alta que hace muy difícil, sino imposible a una escala de tiempo corta, establecer modelos hidrológicos generales para los ríos, cualquier aspecto del medio físico, químico, biológico, funcional o metabólico seguirá una pauta similar. Predecimos que la estructura y el funcionamiento de los ríos de las regiones áridas y semiáridas, deben estar marcadas por la "historia hidrológica anterior" del sistema.

Así pues, el objetivo principal de este trabajo de investigación es analizar la respuesta de distintas variables del medio físico, químico, biológico y funcional, en situaciones hidrológicas extremas, en ríos del sureste peninsular de características ambientales áridas.

\section{METODOLOGÍA}

Los datos utilizados para elaborar el modelo propuesto provienen de distintos estudios llevados a cabo en ríos y ramblas de la cuenca del Segura (Ortega et al., 1991a y b), pero fundamentalmente está basado en los trabajos realizados en el Río Chícamo (Martínez et al., 1998; Suárez \& Vidal-Abarca, 2000; Vidal-Abarca et al., 2000; 2001; 2002; 2004; Velasco et al., 2003; Guerrero et al., 2004; 2006), uno de sus afluentes localizado en el sector más árido del sureste (Vidal-Abarca et al., 1987).
El Río Chícamo es el último afluente del Segura por la márgen izquierda. Se localiza en el sector más árido de sureste ibérico (temperatura media anual $=18^{\circ} \mathrm{C}$; precipitación anual inferior a $300 \mathrm{~mm})$. Se trata de un río intermitente que drena una cuenca de $501.8 \mathrm{~km}^{2}$. De los $60 \mathrm{~km}$ de longitud total del cauce principal, solo transportan agua unos $13.5 \mathrm{~km}(5.8 \mathrm{~km}$ con flujo permanente y un caudal medio de $171 \mathrm{~s}^{-1}$ y $7.7 \mathrm{~km}$ con flujo temporal y un caudal entre $0-0.41 \mathrm{~s}^{-1}$ ).

La litología de la cuenca de drenaje del Río Chícamo esta dominada por calizas y dolomías en el sector topográficamente más alto y por margas miocénicas del Keuper y materiales del Cuaternario en los más bajos. En consecuencia el tramo medio-bajo del Chícamo es salino

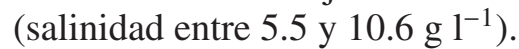

En general, la cuenca del Río Chícamo está cubierta por un matorral típico mediterráneo, aunque en el sector medio-bajo aparecen cultivos de almendro y olivo.

En cuanto al cauce, la vegetación ribereña es, en general, muy escasa diferenciando el sector más alto, donde dominan los helófitos Arundo donax, Phragmites australis, Nerium oleander, Juncus maritimus, Scirpus holochaenus y Tamarix boveana entre otros, del más bajo con especies típicamente halófilas (Arthrocnemun macrostachyum, Sarcocornia fruticosa, Limonium $s p$.). La vegetación sumergida es muy escasa, apareciendo algunos parches de Chara y Enteromorpha intestinalis, siendo dominante la comunidad de perifiton (epipelon).

Los datos utilizados del Río Chícamo provienen de los muestreos realizados desde 1994 hasta 2003 con distinta periodicidad, según el objetivo planteado. Todas las muestras se recogían en un tramo de $350 \mathrm{~m}$ en 10 puntos de muestreos diferentes y siempre por triplicado. Los métodos de recogida y análisis de los parámetros físico-químicos se describen en Vidal-Abarca et al. (2000). Para realizar el cálculo de la superficie inundada, el tramo de estudio fue cartografiado sistemáticamente. La elaboración de las cartografías se realizó mediante el Programa AutoCAD 14 (Versión Española), que permite además, obtener datos morfométricos (superficie, perímetro, etc).

La recogida de muestras de materia orgánica y 
de perifiton (epipelon) y su posterior procesado se describen en Martínez et al. (1998), Vidal-Abarca et al. (2001; 2004) y Velasco et al. (2003). La recogida y procesado de las muestras de invertebrados acuáticos se describe en Guerrero et al. (2004).

\section{RESULTADOS}

\section{Generalidades sobre el cuadro hidroquímico}

En general y según los datos obtenidos, en los ríos mediterráneos semiáridos el verano es hidrológicamente la fase del ciclo anual más predecible. En estas circunstancias la saturación de oxígeno, la temperatura del agua y en definitiva, los procesos de descomposición de la materia orgánica son los que definen el patrón hidroquímico dominante (Vidal-Abarca et al., 2000; 2004). El otoño, por el contrario, es la fase menos predecible. El caudal es, en este caso, el factor más importante. El retardo y, sobre todo la intensidad de las lluvias, marca la diferencia entre periodos donde el agua contiene altas concentraciones de sales, como consecuencia de la redisolución de las mismas por lluvias de poca intensidad (crecidas) (Kilham, 1990), frente a otros periodos donde la concentración de nitratos (provenientes del lavado de los suelos agrícolas y de las margas sedimentarias de la cuenca: Vidal-Abarca et al., 2004) y de los sólidos en suspensión (provenientes del lavado de suelos poco cohesionados), denuncian la existencia de intensas precipitaciones (avenidas) (Vidal-Abarca et al., 2000).

En invierno, tanto la salinidad como la concentración de oxigeno disuelto vuelven a adquirir importancia en el cuadro hidroquímico, dependiendo sobre todo de la cantidad de lluvias caídas durante el otoño. La primavera puede ser una prolongación del invierno, sobre todo cuando no es muy lluviosa. De hecho, la concentración de oxígeno en el agua, es el parámetro hidroquímico más importante, pasando el caudal a segundo término. En definitiva, el cuadro hidroquímico en cada fase de ciclo hidrológico parece depender de la "historia hidrológica" inmediata anterior, como predecíamos.

\section{Las perturbaciones hidrológicas y sus efectos sobre los parámetros físicos, químicos y biológicos}

De todas las perturbaciones naturales que sufren los ríos mediterráneos, las riadas y las sequías se encuentran entre las más habituales e impactantes. De las primeras existe una vasta bibliografía que hace referencia a sus efectos sobre el medio físico, físico-químico, sobre las comunidades biológicas, e incluso sobre parámetros metabólicos y de producción (e.g. Fisher, 1990; Gagneur, 1994; Cherifi \& Loudiki, 1999; Camarasa \& Segura, 2001; Biggs \& Smith, 2002; Uehlinger et al., 2003). Para el caso de las sequías, la información es mucho menos abundante y confusa (e.g. Dodds et al., 1996; Jacobsen \& Encalada, 1998; Meyer \& Meyer, 2000; Schellenberg et al., 2001; Wright et al., 2002; Dahm et al., 2003; Suren et al., 2003; Acuña et al., 2005), pero sin embargo, cada vez se entienden mejor los efectos y las consecuencias de estas situaciones extraordinarias sobre las variables ambientales y procesos ecológicos analizados.

Recientemente, Lake $(2000 ; 2003)$ ha llamado la atención sobre la idoneidad de distinguir entre las causas (es decir las perturbaciones en sí) y los efectos (es decir, la respuesta de las variables a estas perturbaciones) en relación con el tiempo. Se distinguen así, las causas y efectos que se producen a manera de "pulso", "presión" o "rampa" (Fig. 2). A manera de "pulso" actúan las perturbaciones que suceden en un tiempo muy corto $\mathrm{y}$ de forma muy intensa, por ello producen un pico muy agudo (Fig. 2 A, a). Como "presión" actúan las perturbaciones que, al principio generan un pico importante pero después alcanzan un nivel constante que se mantiene (Fig. 2 A, b). Finalmente, la "rampa" describe una perturbación que se incrementa paulatinamente en el tiempo sin un fin o alcanzando una asíntota transcurrido un periodo más largo (Fig. 2 A, c). Las respuestas se producen en este mismo sentido (Fig. 2 B) y el modo en que ocurre depende del nivel de organización sobre el que se trabaja (población, comunidad, ecosistema).

Típicamente una avenida de agua es una perturbación a modo de "pulso" y las sequías se 

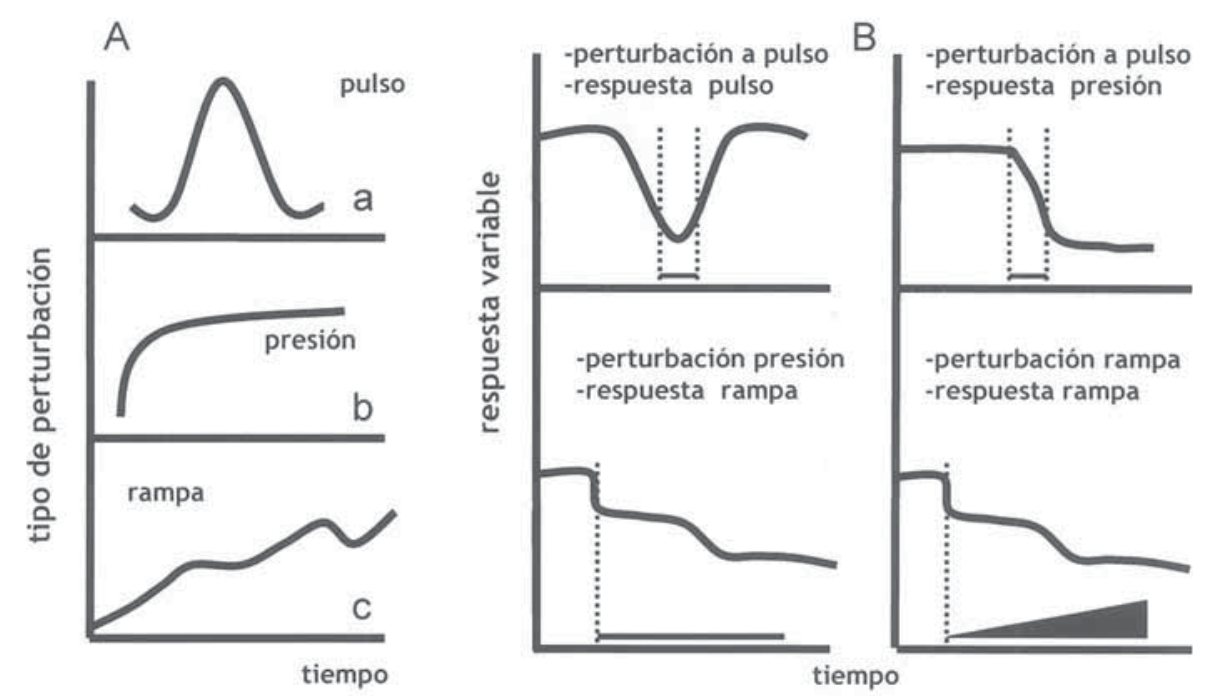

Figura 2. Tipos de perturbaciones y de respuestas en función de su tendencia temporal (redibujado de Lake, 2000). Types of disturbances and responses in relation to temporal tendency (redrawn from Lake, 2000).

comportan a manera de "rampa", pero ¿cómo es la respuesta de las variables físicas, químicas o biológicas a estas perturbaciones?. Tradicionalmente, la ecología se ha fundamentado en la percepción de que las propiedades de un ecosistema (por ejemplo, diversidad, o productividad) cambian de manera suave (gradual) tal como lo hacen las condiciones ambientales (Schmitz, 2004). Recientemente, sin embargo, las teorías emergentes apuntan hacia la posibilidad de que los cambios ambientales pueden producir cambios muy bruscos en las propiedades de los ecosistemas (para ecosistemas acuáticos como lagos y pequeños humedales ver Straile, 2002; Chase, 2003).

A pesar de la escasez de datos e información, en general, existen predicciones sobre las respuestas de los parámetros analizados a estas perturbaciones. Así, Boulton \& Brock (1999), predicen los cambios en varios parámetros físicos, químicos y de las comunidades biológicas, basándose en los trabajos de distintos autores (Stanley \& Boulton, 1995; Baldwin, 1996; Michell \& Baldwin, 1998; McComb \& Qui, 1998, para parámetros físico-químicos; Boulton, 1989, Boulton \& Suter, 1986; Boulton \& Lake 1992b y c; Stanley et al., 1994; Boulton \& Stanley, 1995 para organismos acuáticos). Para los efectos sobre aspectos metabólicos y funcionales ver por ejemplo, Fisher et al., 1982; Meyer et al. (2001); Uehlinger et al. (2003) y Pinna \& Basset (2004).

En las figuras 3 y 4 se presenta, a modo de ejemplo, la respuesta de distintos parámetros físicos, químicos y biológicos medidos en el Río Chícamo, a una crecida producida tras las lluvias en primavera de 1998 (13-14 de mayo) y a un estiaje en el verano de 1999 (desde junio a septiembre) incluyendo la restitución del flujo de agua tras las primeras lluvias de septiembre (3-4 de septiembre de 1999). El muestreo de referencia corresponde a marzo de 1998.

La crecida de primavera es una perturbación de tipo "pulso" y todas las variables analizadas responden también a manera de "pulso", aunque su comportamiento posterior es más complejo, fundamentalmente en el caso de los nutrientes (Fig. 3). El caudal y la anchura de la lámina de agua muestran un fuerte aumento mientras que, la salinidad y la conductividad sufren una disminución como consecuencia del efecto de dilución. El fósforo reactivo soluble aumenta en respuesta, probablemente, a la entrada de materiales orgánicos desde la cuenca de drenaje. Igual comportamiento presenta el amonio, pero su evolución posterior es mucho más compleja, al igual que ocurre con el oxigeno disuelto y los nitratos que, aunque la respuesta inmediata es una 

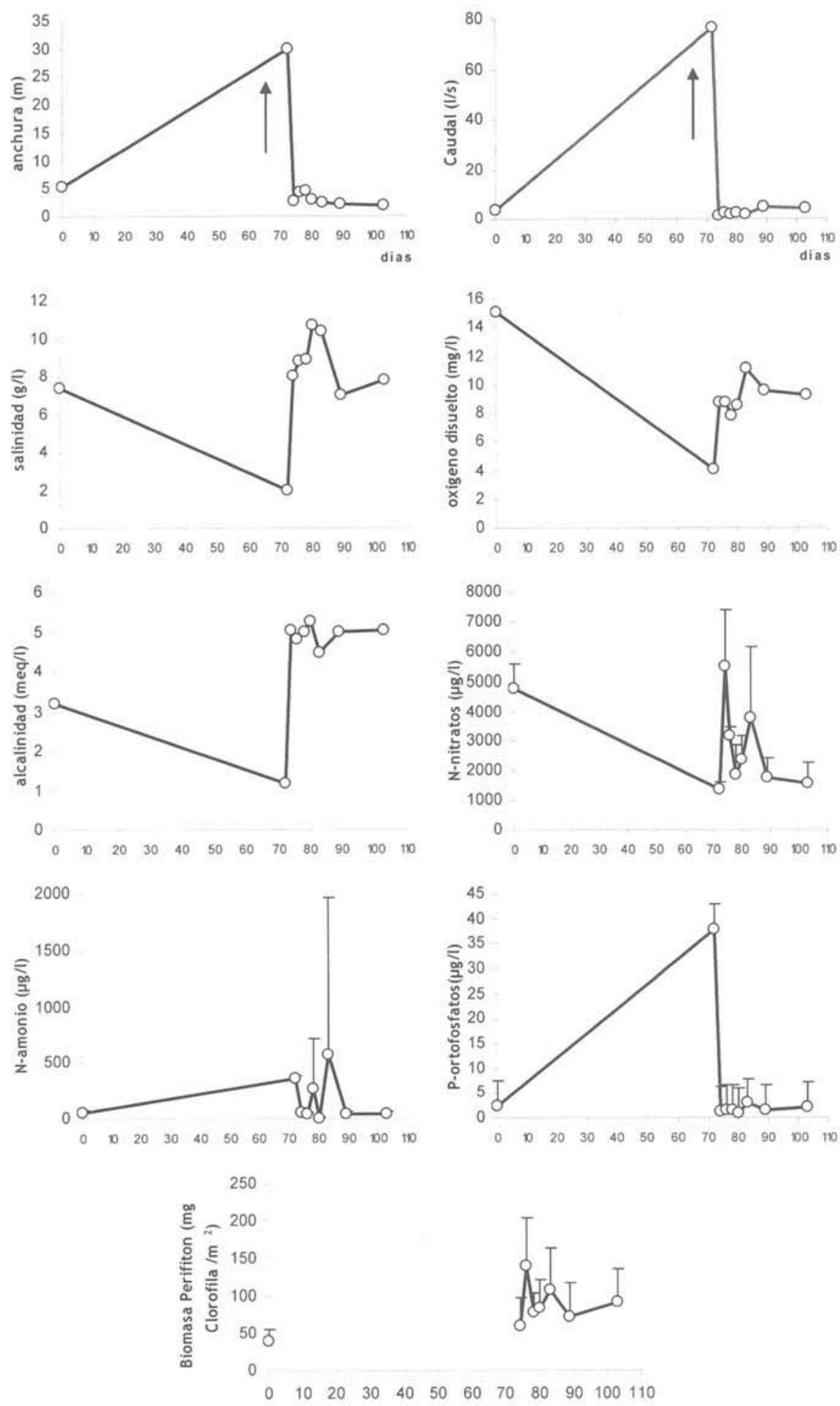

Figura 3. Evolución de los parámetros físicos, químicos y biológicos analizados en el río Chícamo tras la crecida de primavera en mayo de 1998 (El muestreo de referencia es del 5-03-1998). Evolution of the physical, chemical, and biological parameters analyzed in the Chicamo river after the flood in Spring, May of 1998. (Reference sampling is from 5-03-1998). 
disminución drástica de su concentración en el agua, su posterior estabilización debe estar ligada a procesos biológicos como el crecimiento y degradación del perifiton que sigue una pauta similar a estos parámetros (Uehlinger et al., 2003).

El periodo de estiaje que se muestra en la figura 4 no supuso una pérdida total del agua, y se manifiesta como una perturbación a manera de "rampa" aunque la respuesta de los parámetros analizados es más diversa que en el caso anterior. La salinidad y la conductividad no muestran el aumento predicho (Boulton \& Brock, 1999), quizás porque al hacerlo también la temperatura y disminuir la lámina de agua ocurre un proceso de cristalización secuencial de sales. A manera de "rampa" fueron las respuestas de la temperatura del agua, el oxígeno disuelto y la materia orgánica bentónica total (MOB), en este caso aumentando progresivamente sus concentraciones conforme progresa el estiaje. La concentración de nitratos y de amonio, por el contrario, muestran una reducción muy importante, quizás debida a la desnitrificación (más que probable) en el primer caso y a la utilización del amonio por parte de la comunidad perifítica, en el segundo.

El fósforo reactivo soluble, responde a manera "pulso", pero su comportamiento posterior, incluida la respuesta a las lluvias que finalizan el proceso de estiaje, es complejo y parece seguir el modelo propuesto por McComb \& Qui, (1998) para humedales temporales. En dicho modelo, además de los procesos químicos de adsorción a los sedimentos durante la sequía y su posterior liberación tras las lluvias, con el consiguiente rehumedecimiento de los sedimentos, intervienen las bacterias aerobias y anaerobias. La respuesta del perifiton aparece ligada a la del fósforo, de manera que su biomasa aumenta o disminuye en función de la disponibilidad o no de fósforo reactivo soluble en el medio.

El estiaje finaliza tras las lluvias de principios de septiembre, de manera que es posible analizar también la respuesta de estos parámetros a la crecida provocada por las lluvias de otoño. Como se observa en la figura 4, en todos los casos, salvo la materia orgánica bentónica total (MOB) cuya respuesta a las lluvias es a modo de presión, el resto responde a manera de "pulso", aunque en el caso de los nutrientes, la respuesta no es similar a la detectada en la crecida de primavera (Fig. 3). Así, la concentración de nitratos, que tras las lluvias de primavera disminuía considerablemente, ahora experimenta un importante aumento. En cuanto al fósforo, si la respuesta tras la primera crecida era un aumento importante a manera de pulso, tras la sequía se observa también un aumento pero mucho menos importante que en el caso anterior.

En definitiva, la respuesta de las variables analizadas durante el estiaje es mucho más diversa que en el caso de las crecidas, lo cual significa que este tipo de extremos hidrológicos, aún cuando han recibido menos atención, deben implicar cambios importantes en la estructura $\mathrm{y}$ el funcionamiento de los ecosistemas que lo padecen, al menos a escala local. Las crecidas, por el contrario, uniformizan el sistema, aunque la respuesta de las variables puede ser diferente dependiendo de la época hidrológica en la que ocurre. Así, en este caso, parece que nuevamente la "historia hidrológica" anterior a la perturbación, es la clave para entender los procesos que ocurren en estos ecosistemas.

\section{La respuesta de los invertebrados acuáticos a las perturbaciones}

Aunque para las avenidas, existe, en la actualidad, un importante número de trabajos relativo a los efectos sobre los organismos acuáticos y sus adaptaciones para tolerar estas perturbaciones, en el caso de la respuesta de los organismos acuáticos a las sequías, la información es mucho más escasa. Una reciente y completa revisión para peces, se encuentra en Magoulick \& Kobza (2003). Para invertebrados acuáticos las revisiones de Lake (2003), Boulton (2003) y Lytle \& Poff (2004), recogen los estudios realizados hasta la actualidad. Destacar el libro de Williams (2006) sobre aguas temporales.

En cuanto a las avenidas de agua, el efecto sobre los organismos acuáticos suele ser muy drástico disminuyendo tanto la riqueza específica como la abundancia (Fisher et al., 1982; Ortega et al., 1991a). Pero mucho más interesante y diverso 

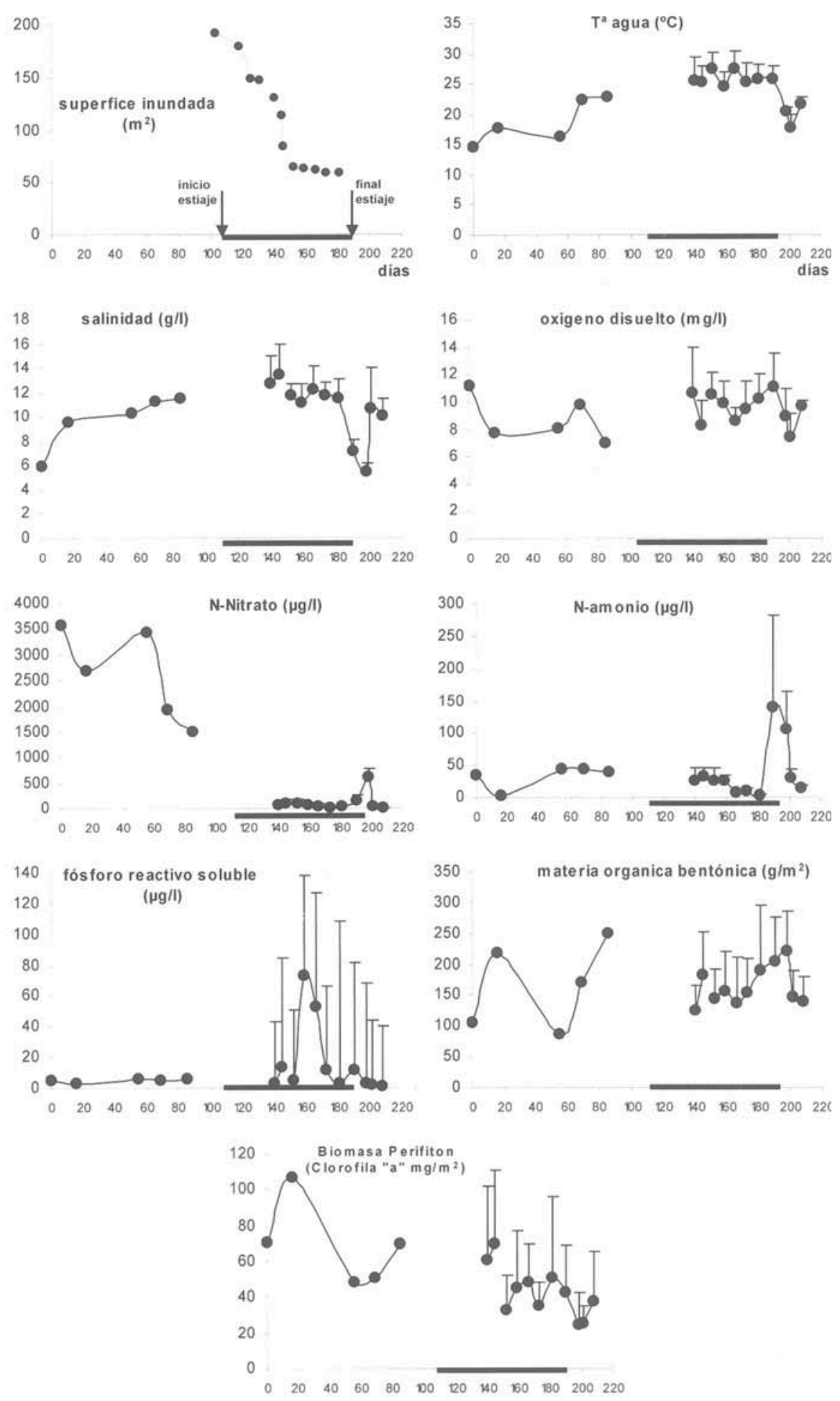

Figura 4. Evolución de los parámetros físicos, químicos y biológicos analizados en el río Chicamo, durante el estiaje de 1999 y posterior recuperación tras las lluvias de septiembre. Evolution of the physical, chemical, and biological parameters analyzed in the Chicamo river during the drought of 1999 and after the recovery due to the September rainfalls. 

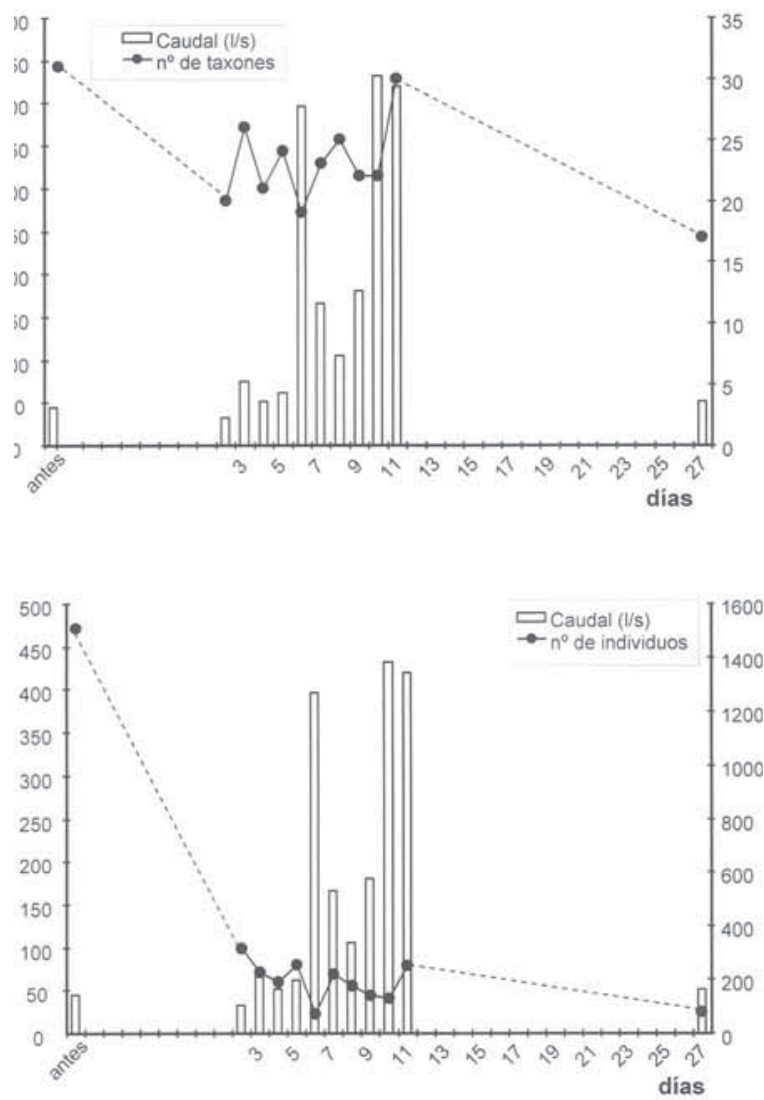

Figura 5. Variación de número de taxones y abundancia de la comunidad de invertebrados acuáticos de la Rambla del Moro tras una avenida de agua. Se indica la evolución del caudal. (Extraído de Ortega et al., 1991 b). Variation of the number of taxa and abundance of the aquatic invertebrate community of the Rambla del Moro after a flash-flood. Evolution of the discharge is shown. (From Ortega et al., 1991 b)

es la secuencia de colonización que se produce posteriormente y que avala la capacidad de resiliencia del sistema, en general (Fisher \& Grimm, 1988, Badri et al., 1987; Ortega et al., 1991b).

Para el caso de las sequías, Lake (2003) secuencia el proceso en tres fases diferentes. En una primera, el sistema se fragmenta, lo cual lleva al aislamiento de los organismos acuáticos, una segunda fase donde se reduce la cantidad de agua y, por tanto el tamaño del habitat, lo que lleva a un aumento relativo de la densidad de los organismos acuáticos y finalmente, si se seca completamente el cauce, a la muerte de la totalidad de los individuos. Además, uno de los efectos más importante parece ser la alteración de
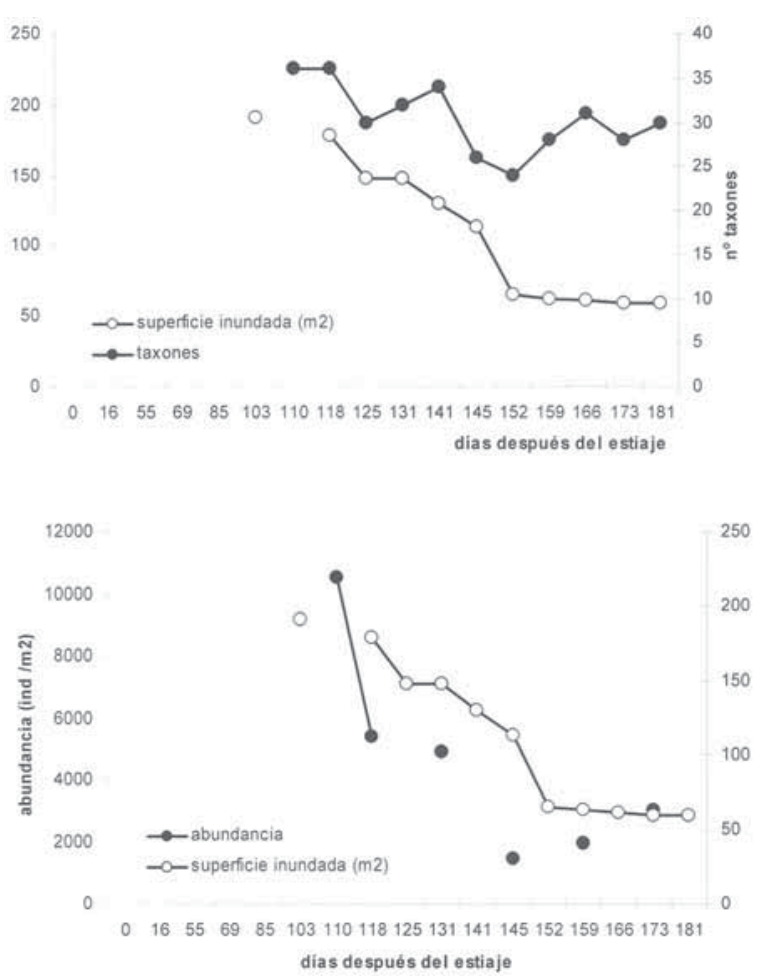

Figura 6. Variación de número de taxones y abundancia de la comunidad de invertebrados acuáticos del Río Chicamo durante el estiaje de 1999. Variation of the number of taxa and abundance of the aquatic invertebrate community of the Chicamo river during the drought of 1999.

la estructura trófica de la comunidad (Boulton \& Lake, 1990, 1992 a y b; Maamri et al., 1997). Boulton (2003), propone una secuencia de cambios en la riqueza y composición de la comunidad de invertebrados acuáticos, durante este proceso, en cuatro fases, donde incluye el papel como "área refugio" que supone la zona hiporreica.

En la figura 5, se presenta la respuesta de la comunidad de invertebrados acuáticos de la Rambla del Moro a una avenida de agua (Ortega et al., 1991 a y b). Como se observa, la comunidad de invertebrados acuáticos sufre una reducción en la riqueza específica, aunque mucho mayor es en relación a la abundancia, donde se observa un efecto de "lavado" importante. La respuesta posterior es rápida para la riqueza, pero no lo es para la abundancia (Fig. 5).

Los efectos de la sequía sobre la riqueza de invertebrados se han analizado en el Río Chícamo (Fig. 6). Como se observa la respuesta es a modo 


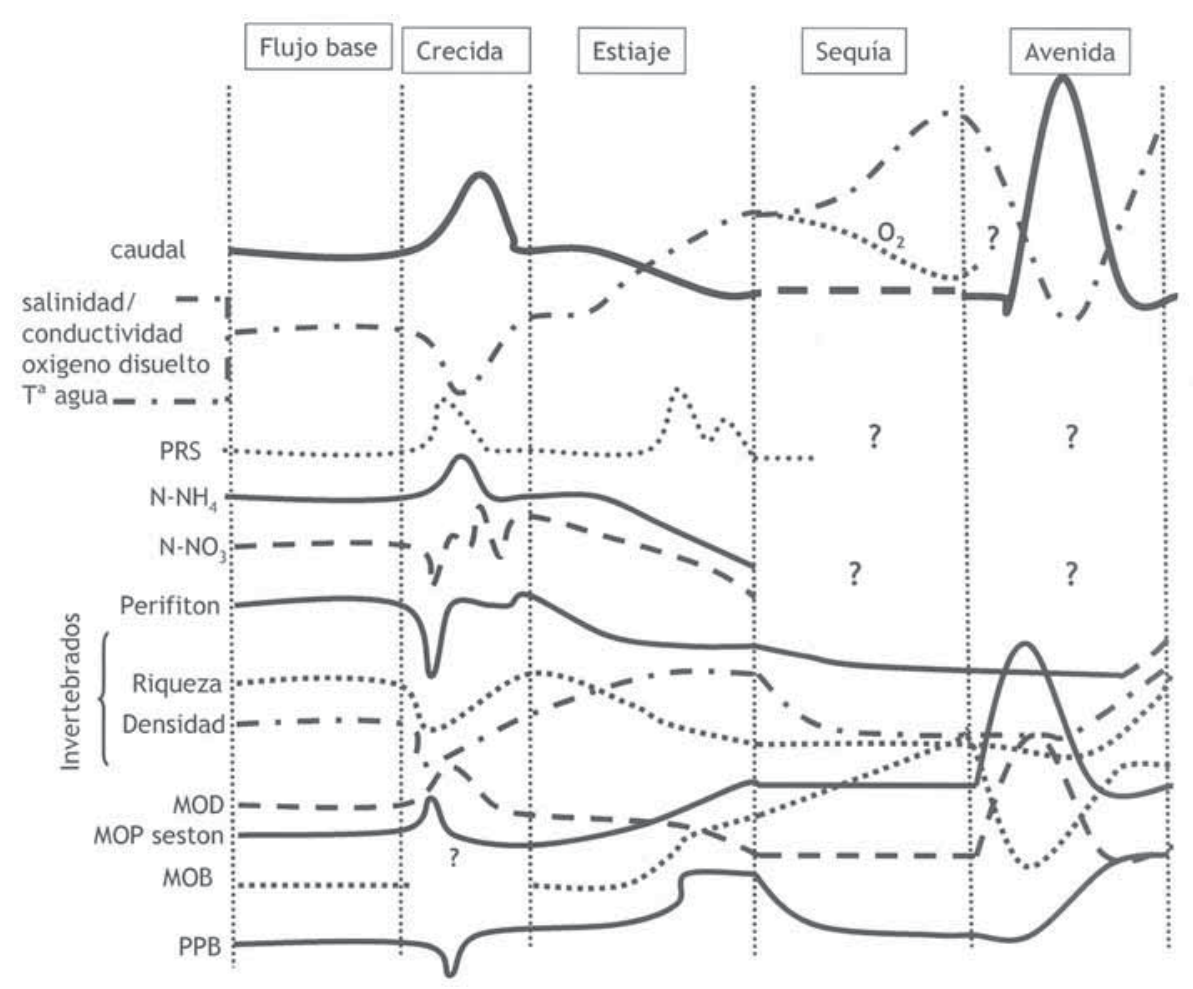

Figura 7. Diagrama representando las distintas fases del ciclo hidrológico anual y las principales perturbaciones hidrológicas (avenidas y sequías) y la respuesta predecible de diferentes parámetros físicos, físico-químicos, biológicos y metabólicos en ríos mediterráneos. Diagram representing the different phases of the annual hydrological cycle and the main hydrological disturbances (floods and droughts) and the predictable response of different physical, physico-chemical, biological, and metabolic parameters in Mediterranean rivers.

de "rampa" como era de esperar, pero llega un momento en el que, aunque persiste la pérdida de agua, no implica una disminución drástica de las especies, lo cual puede significar que las especies que persisten son, precisamente, las más tolerantes a este proceso. No se puede hacer una interpretación similar a la de Boulton (2003) referida al papel de refugio temporal de la zona hiporreica, porque en el Río Chícamo no existe tal zona. El sustrato duro de margas impermeables es la base física del cauce sobre la que se dispone abundante material orgánico, pero impide la existencia de una zona hiporreica porosa. Los datos de abundancia siguen una pauta diferente, dado que al disminuir la superficie inundada aumenta la densidad de individuos, lo cual apoya la hipótesis sobre el efecto de "concentración" que supone la disminución del habitat acuático (Boulton, 2003).
La sustitución de especies, o cambios en la composición de la comunidad que se observan en el transcurso del proceso de sequía, es otro de los principales efectos sobre los que inciden algunos autores (por ejemplo, Boulton, 2003). En el Río Chícamo tal situación no ocurre, de hecho el efecto consiste en una simplificación de la comunidad, que durante este proceso sufre la pérdida de varios taxones, precisamente los más exigentes a condiciones de flujo de agua (por ejemplo, el caso de los simúlidos), incluyendo a especies que requieren además que el flujo discurra sobre sustrato duro (por ejemplo, el caso del molusco Theodoxus fluviatilis) o especies depredadoras que requieren unos recursos, que quedan muy limitados (por ejemplo, los heterópteros Nepa cinera y Naucoris maculatus u odonatos como Crocothemis eryhtraea y Coenagrion scitulum). Cambios en la estructura trófica de la co- 
munidad de invertebrados acuáticos, también han sido documentados en la bibliografía (Boulton \& Lake, 1992a y b; Stanley et al., 1994).

\section{Propuesta de un modelo conceptual para ríos mediterráneos}

A manera de síntesis se ha elaborado la figura 7 donde se presenta la respuesta y tendencias de distintos parámetros físicos, químicos, biológicos y metabólicos, a distintas fases del ciclo hidrológico, en un intento de establecer un marco conceptual para ríos mediterráneos de regiones áridas y semiáridas.

La hidrología, es el parámetro al que responden, de una $u$ otra manera, todas las variables analizadas. Así, las columnas de la figura 7 representan cada una de las fases del ciclo hidrológico que podemos encontrar en el ámbito mediterráneo. El flujo de base se refiere a la situación de máxima "estabilidad" del sistema en la que fluye una cantidad de agua que depende del balance hídrico anual. Las crecidas se producen, de forma bastante predecible cada otoño, tras las lluvias típicas de esta fase del ciclo anual. Lo que no resulta tan predecible es el momento en el que ocurrirán. El estiaje, al igual que para el caso de las crecidas, es muy predecible en verano, lo que resulta más impredecible es la duración que depende, en este caso, del momento en el que comienzan las lluvias de otoño. Hasta aquí tendríamos el patrón hidrológico natural anual de los ríos mediterráneos. Los dos últimas columnas, se refieren a los dos eventos que alteran significativamente el sistema: las avenidas de agua y las sequías. Ambas son perturbaciones hidrológicas naturales con efectos drásticos, pero mientras que las primeras son muy impredecibles en el ámbito mediterráneo y suponen el lavado y exportación de materiales y nutrientes y la muerte de muchos organismos acuáticos, las sequías presentan un grado de impredecibilidad menor y suponen la mineralización de la materia orgánica y nutrientes e igualmente la muerte por deshidratación de muchos organismos acuáticos (Vidal-Abarca et al., 2004).

Las columnas están colocadas de forma más o menos secuencial, según su ocurrencia a lo largo del ciclo hidrológico, pero la propia variabilidad interanual de estos sistemas (Gasith \& Resh, 1999) permite que las columnas puedan combinarse según otra secuencia cualquiera con el fin elaborar la "historia hidrológica" del sistema en estudio.

Finalmente señalar que la respuesta de los parámetros físicos, físico-químicos, biológicos y metabólicos que se presentan en la figura 7, responden de diferente manera según la hidrología, materializada por el caudal en la figura.

\section{CONCLUSIONES FINALES}

- Aunque la variabilidad hidrológica tiene una gran importancia para explicar la variación de muchos parámetros ambientales en cualquier sistema fluvial, independientemente de su localización, en los ríos mediterráneos de regiones áridas y semiáridas lo es de forma especial, sobre todo porque la respuesta de muchos de estos parámetros son consecuencia de la secuencia de eventos a los que están sometidos, o lo que es lo mismo, de la "historia hidrológica" del ecosistema.

- Si las propuestas conceptuales que distintos autores han ido elaborando, han ayudado a configurar las líneas de investigación actuales de la Limnología mundial, la importancia de considerar las escalas en el espacio y en el tiempo, quizás sea la más útil para entender, en toda su dimensión la dinámica de estos ecosistemas acuáticos.

- Falta aún información para completar el "cuadro" final que marque las pautas para establecer la correcta gestión de estos ecosistemas acuáticos singulares. El tiempo es, quizás el parámetro más importante y también el que juega en contra para gestionar de forma sostenible estos ecosistemas difíciles de entender y aún más de conservar.

- Para la gestión sostenible de estos ecosistemas acuáticos, que implica mantener sus condiciones naturales, es necesario combinar muchos criterios diferentes. En este sen- 
tido, constituye un reto para investigadores y gestores, adecuar los principios generales que emanan de la Directiva Marco del Agua, con el fin de definir las condiciones de referencia de estos ecosistemas acuáticos de indudable singularidad.

\section{AGRADECIMIENTOS}

Los resultados de este estudio han sido financiados por el Proyecto REN2001-0644-C0201/HID, Dirección General de Investigación, Ministerio de Ciencia y Tecnología. Nuestro agradecimiento a todos los que han colaborado de alguna manera con su trabajo en el equipo de investigación.

\section{REFERENCIAS}

ACUÑA, V., I. MUÑOZ, M. OMELLA, F. SABATER, A. GIORGI \& S. SABATER. 2005. Drought and postdrought recovery cycles in an intermittent Mediterranean stream: structural and functional aspects. J. N. Am. Benthol. Soc., 24: 919-933.

BADRI, A., J. GUIDICELLI \& G. PRÉVOT. 1987. Effects d'une crue sur la communauté d'invertebres bentiques d'une riviere méditerranéenne, le Rdat (Maroc). Acta Oecologica. Oecol. Genes., 8(4): 481-500.

BALDWIN, D. S. 1996. The effects of exposure to air and subsequent drying on the phosphate sorption characteristics of sediment from a eutrophic reservoir. Limnol. Oceanogr., 41: 1725-1732.

BIGGS, B. J. F. \& R. A. SMITH. 2002. Taxonomic richness of stream benthic algae: Effects of flood disturbance and nutrients. Limnol. Oceanogr., 47: 1175-1186.

BOULTON, A. J. 1989. Over-summering refuges of aquatic macroinvertebrates in two intermittent streams in central Victoria. Transac. Roy. Soc. South Austr.,113: 23-34.

BOULTON, A. J. 2000. Limnology and conservation of rivers in arid inland Australia. Verh. Internat. Ver. Limnol., 27: 655-660.

BOULTON, A. J. 2003. Parallels and contrasts in the effects of drought on stream macroinvertebrate assemblages. Freshwat. Biol., 48: 1173-1185.
BOULTON, A. J. \& P. J. SUTER. 1986. Ecology of temporary streams-an Australian perspective. In: Limnology in Australia. P. De Deckker \& W. D. Williams (eds.): 313-327. CSIRO/Dr. W. Junk. Melbourne/Dordrecht.

BOULTON, A. J. \& P. S. A. LAKE. 1990. The ecology of two intermittent streams in Victoria, Australia. I. Multivariate analyses of physicochemical features. Freshwat. Biol., 24: 123-141.

BOULTON, A. J. \& P. S. A. LAKE. 1992a. Benthic organic matter and detritivorous macroinvertebrates in two intermittent streams in south-eastern Australia. Hydrobiologia, 241: 107-118.

BOULTON, A. J. \& P. S. A. LAKE. 1992b. The ecology of two intermittent streams in Victoria, Australia. II. Comparisons of faunal composition between habitats, rivers and years. Freshwat. Biol., 27: 99-121.

BOULTON, A. J. \& P. S. A. LAKE. 1992c. The ecology of two intermittent streams in Victoria, Australia. III. Temporal changes in faunal composition. Freshwat. Biol., 27: 123-138.

BOULTON, A. J. \& E. H. STANLEY. 1995. Hyporheic processes during flooding and drying in a Sonoran Desert stream. II. Faunal dynamics. Arch. Hydrobiol., 134: 27-52.

BOULTON, A. J. \& M. A. BROCK. 1999. Australian Freshwater Ecology. Processes and management. Gleneagles Publishing. Glen Osmond (Australia). 300 pp.

CAMARASA, A. M. \& F. SEGURA. 2001. Flood events in Mediterranean ephemeral streams (ramblas) in Valencia region, Spain. Catena, 45: 229249.

CHASE, J. M. 2003. Experimental evidence for alternative stable equilibria in a benthic pond food web. Ecol. Lett., 6: 733-741.

CHERIFI, O. \& M. LOUDIKI. 1999. Flood transport of dissolved and suspended matter in the El Abid river basin (Morocco). Hydrobiologia, 410: 287294.

COMIN, F. A. \& W. D. WILLIAMS. 1994. Parched continents: Our common future? In: Limnology now: A paradigm of planetary problems. Margalef, R. (ed.): 473-527. Elsevier, Sc. B.V. Amsterdam.

CUSHING, C. E. \& J. D. ALLAN. 2001. Stream. Their ecology and life. Academic Press. San Diego. 366 pp.

DAHN, C. N., M. A. BARER, D. I. MOORE \& J. R. THIBAULT. 2003. Coupled biogeochemical and 
hydrological responses of streams and rivers to drought. Freswat. Biol., 48: 1219-1231.

DAVIES, B. R., M. C. THOMS, K. F. WALKER, J. H. O'KEEFFE \& J. A. GORE. 1994. Drylands rivers: Their ecology, conservation and management. In: The river handbook. Callow, P. \& G.E. Petts (eds.): 484-511. Blackwell. Oxford.

DODDS, W. K. 2002. Freshwater Ecology. Concepts and environmental applications. Academic Press. San Diego. 569 pp.

DODDS, W. K., R. E. HUTSON, A. C. EICHEM, M. A. EVANS, D. A. GUDDER, K. M. FRITZ \& L. GRAY. 1996. The relationship of floods, drying, flow and light to primary production and producer biomass in a prairie stream. Hydrobiologia, 333: 151-159.

FIRTH, P. \& S. G. FISHER (eds.). 1992. Global climate change and freshwater ecosystems. SpringerVerlag. New York. 321 pp.

FISHER, S. G. 1990. Recovery processes in lotic ecosystems: limits of succesional theory. Environm. Manag., 14(5): 725-736.

FISHER, S. G., L. J. GRAY, N. B. GRIMM \& D. E. BUSCH. 1982. Temporal succession in a desert stream ecosystem following flash flooding. Ecol. Monogr., 52: 93-110.

FISHER, S. G. \& N. B. GRIMM. 1988. Disturbance as a determinant of structure in a Sonoran Desert stream ecosystem. Verh. Internat. Ver. Limnol., 23: 1183-1189.

FISHER, S. G., N. B. GRIMM, E. MARTÍ, R. M. HOLMES \& J. B. JONES Jr. 1998. Material Spiralling in Stream Corridors: A telescoping Ecosystem Model. Ecosystems, 1: 1-19.

GAGNEUR, J. 1994. Flash-floods and drying up as major disturbances upon benthic communities in North-African wadis. Verh. Internat. Ver. Limnol., 25: 1807-1811.

GASITH, A. \& V. H. RESH. 1999. Streams in Mediterranean Climate Regions: Abiotic Influences and Biotic Responses to Predictable Seasonal Events. Annu. Rev. Ecol. Syst., 30: 51-81.

GARCÍA DE PEDRAZA, L. 1989. Climas de transición al árido. Climas áridos, dominios áridos y semiáridos en España. En: Zonas Aridas en España.: 45-63. Real Academia de Ciencias Exactas, Físicas y Naturales. Madrid.

GILLER, P. S. \& B. MALMQVIST. 1998. The Biology of streams and rivers. Oxford University Press. Oxford. 296 pp.
GRIMM, N. B. \& S. G. FISHER. 1992. Responses of arid-land streams to changing climate. In: Climate, Change and Freshwater ecosystems. P. Firth \& S. G. Fisher (eds.): 211-233. Springer-Verlag. New York.

GUERRERO, C., M. R. VIDAL-ABARCA, M. L. SUÁREZ, R. GÓMEZ y M. ORTEGA. 2004. Estructura de tamaño de la comunidad de macroinvertebrados acuáticos en un río de características semiáridas durante el estiaje (Río Chícamo, SE España). Limnetica, 23 (1-2): 37-56.

GUERRERO, C., M. L. SUÁREZ, y M. R. VIDALABARCA. 2006. Diversidad y heterogeneidad espacio-temporal: Aplicación a la comunidad de invertebrados acuáticos de un río de características semiáridas (SE de España). En: Diversidad Biológica y biodiversidad. (F. García Novo, F. Díaz Pineda \& A. Gómez Sal (coords.): 73-77. Fundación Ramón Areces. Madrid. España.

JACOBSEN, D. \& A. ENCALADA. 1998. The macroinvertebrate fauna of Ecuatorian highland streams in the wet and dry season. Arch. Hydrobiol., 142: 53-70.

JUNK, W. J., P. B. BAYLEY \& R. E. SPARKS. 1989. The flood pulse concept in river-floodplain systems. Can. Sp. Pub. Fish. Aq. Sc., 106: 110-127.

KILHAM, P. 1990. Mechanisms controlling the chemical composition of lakes and rivers: Data from Africa. Limnol. Oceanogr., 35: 80-83.

LAKE, P. S. 2000. Disturbance, patchiness, and diversity in streams. J. N. Am. Benthol. Soc., 19: 573592.

LAKE, P. S. 2003. Ecological effects of perturbation by drought in flowing waters. Freshwat. Biol., 48: 1161-1172.

LIKENS, G. E. 1999. Afterword: Reflections and needs. In: Arid lands management. Toward ecological sustainability. Hoekstra,T. W. \& M. Shachak (eds.): 269-272. University of Illinois Press \& International Arid LandsConsortium. Urbana y Chicago.

LYTLE, D. A. \& N. L. POFF. 2004. Adaptation to natural flow regimes. Trends in Ecology and Evolution, 19: 94-100.

MAAMRI A., H. CHERGUI \& E. PATTEE. 1997. Leaf litter processing in a temporary northeastern Moroccan river. Arch. Hydrobiol., 140: 513-531.

MAGOULICK, D. D. \& R. M. KOBZA. 2003. The role of refugia for fishes during drought: a review and synthesis. Freshwat. Biol., 48: 1186-1198. 
MARTÍNEZ, B., J. VELASCO, M. L. SUÁREZ \& M. R. VIDAL-ABARCA. 1998. Benthic organic matter dynamic in an intermittent stream in Southeast Spain. Arch. Hydrobiol., 141: 303-320.

MCCOMB, A. J. \& S. QUI. 1998. The effects of drying and reflooding on nutrient release from wetland sediments. In: Wetlands in a dry land: Understanding for management. W. D. Williams (ed.): 147-159. Environment Australia Biodiversity Group, Camberra.

MEYER, A. \& E. I. MEYER. 2000. Discharge regime and the effect of drying on macroinvertebrate communities in a temporary karst stream in East Wesphalia (Germany). Aquat. Sci., 62: 216-231.

MEYER, E. I., E. T. SCHELLENBERG \& R. ZAH. 2001. Discontinuities in stream systems induced by low flow and drought. Verh. Internat. Ver. Limnol., 27: 3727-3728.

MICHELL, A. \& D. S. BALDWIN. 1998. The effects of desiccation/oxidation on the potential for bacterially mediated $\mathrm{P}$ release from sediments. Limnol. Oceanogr., 43: 481-487.

MOLINA, C., M. R. VIDAL-ABARCA \& M. L. SUÁREZ. 1994. Floods in arid south-east spanish areas: A historical and environmental review. In: Coping with floods. Rossi, G., N. Harmancioglu \& V. Yevjevich (eds.): 271-278. NATO ASI. Serie E: Applied Sc. Vol. 257. Kluwer Acad. Publ. Netherlands.

ORTEGA. M., M. L. SUÁREZ, M. R. VIDALABARCA \& L. RAMÍREZ-DÍAZ. 1991a. Aspectos dinámicos de la composición y estructura de la comunidad de invertebrados acuáticos de la Rambla del Moro después de una riada (Cuenca del Río Segura: SE. de España). Limnetica, 7: 11-24.

ORTEGA M., M. L. SUÁREZ, M. R. VIDALABARCA, R. GÓMEZ \& L. RAMÍREZDÍAZ. 1991b. Aspects of postflood recolonization of macroinvertebrates in a "Rambla" of South-East Spain ("Rambla del Moro": Segura River Bassin). Verh. Internat. Ver. Limnol., 24: 1994-2001.

PINNA, M. \& A. BASSET. 2004. Summer drought disturbance on plant detritus decomposition processes in three Tiver Tirso (Sardinia, Italy) subbasins. Hydrobiologia, 522: 311-319.

POFF, N. L. 1996. A hydrogeography of unregulated streams in the United States and an examination of scale-dependence in some hydrological descriptors. Freshwat. Biol., 36: 71-91.

SCHELLENBERG, E. T., U. HARTMANN, R. ZAH \& E. I. MEYER. 2001. Response of the epibenthic and hyporheic invertebrates to stream drying in a prealpine river. Verh. Internat. Ver. Limnol., 27: 3733-3737.

SCHMITZ, O. J. 2004. Perturbation and abrupt shift in trophic control of biodiversity and productivity. Ecol. Lett., 7: 403-409.

STANFORD, J. A. \& J. V. WARD. 1993. An ecosystem perspective of alluvial rivers: connectivity and the hyporheic corridor. J. N. Am. Benthol. Soc., 12: 48-60.

STANLEY, E. H. \& S. G. FISHER. 1992. Intermittency, disturbance and stability in stream ecosystem. In: Aquatic ecosystems in semi-arid regions: implications for resource management. Robarts, R. D. \& M. L. Bothwell (eds.): 271-280. National Hydrology Research Institute Symposium Series, 7 , Environment Canada. Saskatoon.

STANLEY, E. H., D. L. BUSCHMAN, A. J. BOULTON, N. B. GRIMM \& S. G. FISHER. 1994. Invertebrate Resistance and Resilience to Intermittency in a Desert Stream. Am. Midl. Nat., 131: 288300.

STANLEY E. H. \& A. J. BOULTON. 1995. Hyporheic processes during flooding and drying in a Sonoran Desert stream. I. Hydrologic and chemical dynamics. Arch. Hydrobiol., 134: 1-26.

STRAILE, D. 2002. North Atlantic oscillation synchronizes food web interactions in central European lakes. Proc. R. Soc. Lond. B., 269: 391-395.

SUÁREZ, M. L. \& M. R. VIDAL-ABARCA. 1993. Las ramblas mediterráneas. Paisajes. En: Regeneración de la cubierta vegetal. Actas V aula de Ecología; Las Ramblas Mediterráneas. Actas VI aula de Ecología. M. Cueto \& A. Pallarés (Coord.): 117-123. Instituto de Estudios almerienses. Diputación de Almería.

SUÁREZ, M. L. \& M. R. VIDAL-ABARCA. 2000. Metabolism in a semiarid stream of south-east Spain. Ver. Internat. Verein. Limnol., 27: 756-761.

SUÁREZ, M. L., M. R. VIDAL-ABARCA, R. GÓMEZ \& L. RAMÍREZ-DÍAZ. 1995. Las avenidas de agua en la configuración de los paisajes de regiones áridas y semiáridas: Consideraciones sobre las obras de control. Actas de las VI Jornadas sobre el Paisaje: Agua y paisaje. Asociación para el Estudio del Paisaje. Segovia (España): 15-26.

SUREN, A. M., B. J. F. BIGGS, M. J. DUNCAN \& L. BERGEY. 2003. Benthic community dynamics during summer low-flows in two rivers of contrasting enrichment 2. Invertebrates. New Zealand J. Mar. Freshw. Res., 37: 71-83. 
UEHLINGER, U., B. KAWECKA \& C. T. ROBINSON. 2003. Effects of experimental floods on periphyton and stream metabolism below a high dam in the Swiss Alps (River Spöl). Aquat. Sci., 65: 199-209.

UYS, M. C. \& J. H. O'KEEFFE. 1997. Simple words and fuzzy zones: Early directions for temporary river research in South Africa. Environm. Manag., 21: 517-531.

VANNOTE, R. L., G. W., MINSHALL, K. W., CUMMINS, R. SEDELL \& C.E. CUSHING. 1980. The river continuum concept. Can. J. Fish. Aq. Sc., 37: 130-137.

VELASCO, J., A. MILLÁN, M. R. VIDAL-ABARCA, M. L. SUÁREZ, C. GUERRERO \& M. ORTEGA. 2003. Macrophytic, epipelic and epilithic primary production in a semiarid mediterranean stream. Freshwat. Biol., 48: 1408-1420.

VIDAL-ABARCA, M. R. 1990. Los ríos de las cuencas áridas y semiáridas: Una perspectiva ecológica comparativa y de síntesis. Scientia gerundensis, 16(1): 219-228.

VIDAL-ABARCA, M. R., C. MONTES, M. L. SUÁREZ \& L. RAMÍREZ-DÍAZ. 1987. El clima de la Cuenca del Río Segura: Factores que lo controlan. Anales de Biología, 12 (Ambiental, 3): 11-28.

VIDAL-ABARCA, M. R., M. L. SUÁREZ \& L. RAMÍREZ-DÍAZ. 1992. Ecology of spanish semiarid streams. Limnetica, 8: 151-160.

VIDAL-ABARCA, M. R., M. L. SUÁREZ \& L. RAMÍREZ-DÍAZ. 1995. Características ambientales y recursos hídricos de la Cuenca del Río Segura. En: Agua y Futuro en la Región de Murcia. M. Senent \& F. Cabezas (eds.): 291-300. Asamblea
Regional de Murcia.

VIDAL-ABARCA, M. R., M. L. SUÁREZ, J. L. MORENO, R. GÓMEZ \& I. SÁNCHEZ. 2000. Hidroquímica de un río de características semiáridas (Río Chícamo; Murcia): Análisis espacio-temporal. Limnetica, 18: 57-73.

VIDAL-ABARCA, M. R., M. L SUÁREZ, C. GUERRERO, J. VELASCO, J. L. MORENO A. MILLÁN \& A. PERÁN. 2001. Dynamics of dissolved and particulate organic carbon in a saline and semiarid stream of southeast Spain (Chícamo stream). Hydrobiologia, 455: 71-78.

VIDAL-ABARCA, M. R., M. L. SUÁREZ, R. GÓMEZ, J. L. MORENO \& C. GUERRERO. 2002. Diel variations in physical and chemical parameters in a semiarid stream of Spain (Chicamo stream). Ver. Internat. Verein. Limnol., 28: 1111-1115.

VIDAL-ABARCA, M. R., R. GÓMEZ \& M. L. SUÁREZ. 2004. Los ríos de las regiones semiáridas. Ecosistemas, 204/1. URL:http//www.aeet. org/ecosistemas/041/revision4.htm/)

WARD, J. V. \& J. A. STANDFORD. 1983. The serial discontinuity concept of lotic ecosystems. In: Dynamics of lotic ecosystems. T. D. Fontaine \& S. M. Bartell (eds.): 29-42. Ann Arbor Science Publisher. Michigan.

WILLIAMS, D. D. 2006. The biology of temporary waters. Oxford University Press. Oxford \& New York. 337 pp.

WRIGHT J. F., R. J. M. GUNN, J. M. WINDER, R. WIGGERS, N. T. KNEEBONE \& R. T. CLARKE. 2002. The impact of drought events in 1976 and 1997 on the macroinvertebrate fauna of a chalk stream. Verh. Internat. Verein. Limnol., 28: 948-952. 